\title{
EL DESAFío De gobernar CIUDADES EN ARGENTINA. ALCANCES Y PERSPECTIVAS EN MATERIA DE ORGANIZACIÓN POLÍTICA Y ADMINISTRATIVA
}

\section{THE CHALLENGE OF GOVERNING CITIES IN ARGENTINA. SCOPE AND PERSPECTIVES FOR POLITICAL AND ADMINISTRATIVE ORGANIZATION}

\author{
Rodrigo Carmona ${ }^{1}$
}

\begin{abstract}
Resumen
El trabajo busca examinar los alcances y perspectivas en materia de organización política y administrativa que presentan los centros urbanos de Argentina en la actualidad, dando cuenta de la heterogeneidad de situaciones, atribuciones y posibilidades de los territorios, además de la fuerte influencia de los otros niveles de gobierno en su desarrollo. Las distintas transformaciones desplegadas en las últimas décadas han puesto en cuestión un modo de gobernar y gestionar ciudades en la Argentina. Procesos heterogéneos tales como la globalización, la reforma del Estado, la desigualdad y la segregación socioespacial al interior de las urbes, el surgimiento de nuevas demandas y reivindicaciones de derechos y el despliegue de nuevas políticas urbanas junto a una mayor intervención del nivel central de gobierno en los años recientes, aparecen como dinámicas que han impactado e impactan sobre los centros urbanos de nuestro país. En estos términos, el desafío de gobernar ciudades que se vuelven más complejas amplía las funciones tradicionales centradas en la provisión de servicios básicos y plantea la necesidad de nuevas respuestas y capacidades ante las demandas que acontecen.
\end{abstract}

Palabras claves: gobierno, organización, ciudades

\section{Abstract}

The paper seeks to examine the scope and prospects for political and administrative organization presenting the urban centers of Argentina today, accounting for the heterogeneity of situations, powers and possibilities of the territories, in addition to the strong influence of the other levels of government in their development. The various transformations deployed in recent decades have questioned a way to govern and manage cities in Argentina. Heterogeneous processes such as globalization, state reform, inequality and spatial segregation with in the cities, the emergence of new rights claims and demands and deployment of new urban policies with greater involvement of the central government level recent years, appear to have impacted dynamics and impact on urban centers of our country. In these terms, the challenge of governing cities become more complex extends the traditional roles focused on the provision of basic services and raises the need for new solutions and capabilities to the demands that occur.

Keywords: government, organization, cities

\footnotetext{
${ }^{1}$ Pos doctor en Ciencias Humanas y Sociales, Facultad de Filosofía y Letras -Universidad de Buenos Aires. Doctor en Ciencias Sociales (Mención honorífica), Facultad Latinoamericana de Ciencias Sociales. Investigador- Instituto del Conurbano. E-mail: rcarmona@ungs.edu.ar
} 


\section{INTRODUCCIÓN}

Las distintas transformaciones desarrolladas en las últimas décadas han puesto en cuestión un modo de gobernar y gestionar ciudades en la Argentina. Procesos heterogéneos tales como la globalización, la reforma del Estado, la desigualdad y segregación socioespacial al interior de las urbes, el surgimiento de nuevas demandas y reivindicaciones de derechos y el despliegue de nuevas políticas urbanas junto a una mayor intervención del nivel central de gobierno en los años recientes, aparecen como dinámicas que han impactado e impactan sobre los centros urbanos de nuestro país.

En este marco, los gobiernos locales han sido testigos de una ampliación de sus funciones y responsabilidades y en menor medida en sus atribuciones, debiendo proveer de este modo un conjunto mayor y más heterogéneo de servicios a la comunidad, sin que ello haya implicado un incremento de sus recursos (económicos, humanos y técnico- administrativos) acorde al crecimiento en la cantidad y complejidad de sus tareas. De este modo, se ha potenciado y en alguna medida resignificado el ámbito local-urbano. Los municipios, como forma administrativa predominante de las ciudades argentinas, han comenzado a tener una mayor importancia en razón de la cercanía dada entre gobernantes y gobernados, posibilitando de este modo el nacimiento gradual de nuevas prácticas y formas organizativas de resolución de problemas. Ello, igualmente, varía y presenta resultados diversos según las particularidades y capacidades de las ciudades en función de su escala, historia y recursos.

En estos términos, el desafío de gobernar ciudades que se vuelven más complejas amplia las funciones tradicionales centradas en la provisión de servicios básicos y plantea la necesidad de nuevas respuestas y capacidades ante las demandas que acontecen. El trabajo busca examinar así los alcances y perspectivas en materia de organización política y administrativa que presentan los centros urbanos de nuestro país en la actualidad, dando cuenta de la heterogeneidad de situaciones, atribuciones y posibilidades de los territorios, además de la fuerte influencia de los otros niveles de gobierno en su desarrollo.

El artículo se organiza del siguiente modo. En primer lugar, se analiza el debate conceptual respecto a nuevas formas de gobierno y gestión pública en el marco de las ciudades y los principales modelos políticos a ser aplicados. En segundo lugar, se examina el escenario argentino y se hace un balance respecto a la organización política y administrativa predominante en los centros urbanos y sus implicancias en términos institucionales, sociales y de planificación territorial. Finalmente, se hacen unas consideraciones finales sobre la problemática y sus desafíos en la coyuntura actual. 


\section{EL DEBATE SOBRE NUEVOS ESTILOS DE GOBIERNO Y GESTIÓN PÚBLICA EN EL MARCO DE LAS CIUDADES}

Las ciudades constituyen a escala planetaria un actor protagónico del capitalismo contemporáneo por su peso demográfico, político, cultural, social y económico. Los procesos simultáneos de globalización, concentración de la población en centros urbanos y descentralización, rediseñan las funciones y el peso específico de estos espacios y los gobiernos locales. En este marco, la ciudad adquiere un rol más preponderante y se transforma fuertemente por las diferentes dinámicas estructurales en juego. En ellas se despliegan una multiplicidad de interacciones, conflictos y procesos sociales y políticos complejos, con actores que presentan diversas lógicas, capacidades e intereses. La conformación del espacio urbano como una instancia que aglutina dinámicas variadas y en tensión, que van desde su importancia como ámbito de convivencia, integración y reconocimiento de derechos y al mismo tiempo lugar de fragmentación, segregación e inseguridad, son partes de su mismo desarrollo. Las ciudades componen así una construcción colectiva variada, contradictoria y en permanente transformación.

Más específicamente, la globalización y la emergencia de la esfera local urbana conforman tendencias complementarias. El proceso de globalización económica, cultural y social establece la intensificación de los flujos de información, comercio, conocimiento y relaciones humanas dentro de un sistema donde las ciudades constituyen nodos o centros predominantes en su funcionamiento. Los cambios en los patrones de localización de las empresas, la menor preponderancia de los Estados-nación, el aumento de la población urbana y las mayores posibilidades de comunicación global establecen un cuadro caracterizado por el incremento de la competencia y la complejidad entre ciudades y regiones, y a la vez mayores diferencias y desigualdades a escala territorial y local (Castells, 1996; Borja y Castells, 1997; Sassen, 1994 y 1999).

Las aglomeraciones urbanas y metrópolis son entonces el resultado de un proceso de crecimiento económico y demográfico a través de los años que ha generado que la población se concentre donde las actividades y las oportunidades son mayores; esto es, en las ciudades y especialmente en las grandes ciudades. Ellas aparecen como centros de generación de riqueza y dinamismo económico y financiero dentro de una lógica de funcionamiento a escala global. Del mismo modo, son la expresión de nuevos conflictos y problemas vinculados a la pobreza urbana (desigualdad, segregación socioespacial, precarización laboral) como también, laboratorios de experimentación respecto a modalidades novedosas en las 
formas de hacer política y la participación democrática (Graña, 2005, Pírez, 2008).

La reforma del Estado que se desarrolló fuertemente en América Latina durante los años '90, reposicionó a la ciudad a partir de la descentralización y los procesos de apertura y delegación "hacia abajo" de las funciones de gobierno por las medidas de ajuste y austeridad del nivel central. Ello sumado a la globalización y la concentración demográfica, determinaron el protagonismo de las ciudades en la actualidad.

En efecto, América Latina conforma hoy en día un continente plenamente urbano. Según el Informe ONU- Estado de la Población Mundial 2011, el 79\% promedio de la población habita en ciudades y se calcula que en 2050 lo hará en su conjunto el $90 \%$. Ello muestra una tendencia general expresada en importantes concentraciones poblaciones en los núcleos urbanos grandes (megalópolis de más de 10 millones de habitantes, como México, San Pablo Buenos Aires y Río de Janeiro) y en ciudades que se metropolizan (46 urbes con más de un millón de habitantes). Es posible apreciar también, el redireccionamiento de flujos migratorios hacia nuevos sitios -del exterior inclusive, dentro de una lógica de conexión global-, el crecimiento de las periferias de las grandes ciudades y de ciertas áreas dinámicas al interior de los países.

Las ciudades emergentes y las principales aglomeraciones urbanas con proyección regional -generalmente, capitales nacionales- constituyen así el eje más dinámico del proceso, con acciones más integradas o competitivas según el caso. En este marco, tal como resalta Carrión (2007), es posible apreciar distintos modelos políticos de gobierno de la ciudad. Un modelo remite a un enfoque mercantil privado y concibe al manejo público-estatal como un limitante para el desarrollo urbano, mientras que el otro le asigna un mayor peso a lo ciudadano con eje en un enfoque de derechos. Estos dos modelos de gobierno urbano no se muestran de manera pura, aunque puede apreciarse el predominio de uno sobre el otro. El primero es el de la ciudad empresarial-privada, como mecanismo que difunde y generaliza los principios de mercado en un contexto de demanda disgregada y atomización de los conflictos urbanos. Se critica la intervención estatal por ineficiente y centralizadora de las decisiones, frente a los beneficios delo privado en términos de eficiencia y mayor participación de la ciudadanía pensada en forma privatista y desde una lógica de "cliente". En el plano administrativo, con un alcalde pensado como gerente se propone una reestructuración del aparato municipal mediante tercerizaciones de los servicios y beneficios a sectores específicos. Esta dinámica privilegia la gestión privada sobre la pública, y modifica además la relación entre el Ejecutivo y el concejo municipal, lo cual perjudica la representación y una visión integral del conjunto al concentrar las decisiones en una fracción alejada de la sociedad. De este modo, se busca 
«despolitizar» las decisiones relevantes de política urbana otorgando mayor injerencia a grupos privados de modo de lograr un manejo presupuestario más eficiente y dotar de mayor gobernabilidad a la gestión municipal

El otro modelo alternativo, que el autor denomina «ciudad inclusiva», busca resignificar lo público como una opción frente a los problemas urbanos mediante distintas estrategias. En primer término, sobre la base del restablecimiento del aparato municipal pensado como instancia amplia de gobierno, dentro del cual la participación y la representación son elementos centrales. Por otra parte, a partir de la reconstitución de la ciudad como espacio público pensado como factor estructurante de la ciudad tanto en su dimensión física (organización espacial), social (identidades e integración) y ciudadana (constructor de derechos: salud, educación, etc.). Se destacan así las ideas de democratización del gobierno local y de racionalización de su administración pública poniéndose el énfasis en lo territorial por sobre lo sectorial. Este enfoque busca afianzar la gobernabilidad y el desarrollo urbano (económico, social, cultural), mediante una mejor integración social y politización de su funcionamiento.

En estos términos, en el debate sobre políticas urbanas es posible identificar distintas tendencias de cambio e innovación. En primer lugar, frente a las políticas de carácter homogéneo y generalista en el tratamiento de los problemas urbanos se resalta la importancia puesta en reconocer las especificidades de los territorios de modo de adaptar las agendas y las formas de intervención a las particularidades de cada lugar. Al mismo tiempo, se enfatiza en la voluntad de propiciar procesos de transformación sobre la base de un enfoque estratégico que combine diagnóstico, prospectiva y actuaciones desde una lógica de tipo transversal e intersectorial. Se pone el acento también en el despliegue de redes e interacciones entre los múltiples actores -gubernamentales y sociales- involucrados en el territorio. Estos aspectos resaltan así una nueva concepción de política urbana centrada en fortalecer los elementos de proximidad y de participación de los actores implicados (Blanco y Subirats, 2008; Cabrero Mendoza, 2005 y Carmona, 2012).

Estos procesos en la literatura especializada pueden circunscribirse a la noción de gobernanza, que en líneas generales plantea al pasaje gradual de una modalidad de actuación gubernamental cuyo eje está en la primacía excluyente del gobernante en el plano de la dirección y conducción política, a una situación donde se establecen mayores grados de interacción y dependencias mutuas entre las instancias político-institucionales y sociales (Blanco y Gomá, 2006; Aguilar Vilanueva, 2007). De este modo, la aplicación básica que identificamos a partir del concepto en el marco de las ciudades es la decisión política de parte de las autoridades locales, más o menos voluntaria, de compartir el poder, de democratizar las 
decisiones de gobierno, incorporando a actores no estales, que se espera representen lo más ampliamente posible los distintos intereses sociales, en el diseño, implementación, evaluación y control de las acciones gubernamentales.

Por otra parte, las ciudades en su proceso de expansión incluyen territorios diversos y delimitados en el plano político-institucional. Un primer nivel se corresponde con el ámbito jurisdiccional más próximo, que es el gobierno municipal o regional en algunas circunstancias. Sin embargo, las ciudades en su despliegue pueden desbordar estos límites y en ciertos casos implicar áreas superiores que involucran diversos gobiernos municipales $\mathrm{y} / \mathrm{o}$ regionales. Si observamos la problemática de la gobernabilidad urbana como la capacidad del gobierno de la ciudad de orientar y conducir los grandes procesos urbanos de modo de responder a las necesidades de la población y las actividades económicas, en una ciudad metropolitana ella se relaciona a la importancia de una perspectiva global que dé cuenta de la "ciudad real" en términos de un ámbito gubernamental específico (Pírez, 2001 y 2004).

Al analizar estas problemáticas y situarnos en la realidad latinoamericana, contemplamos las dificultades de efectivizar un "gobierno de la ciudad real" o gobierno metropolitano. El municipio constituye generalmente la instancia institucional gubernamental central de la ciudad, la cual debe responder con capacidades y recursos al límite a los distintos requerimientos que la nueva escena urbana depara. En los últimos años, esta escala como también la regional gana protagonismo político producto de los procesos de índole estructural examinados y el despliegue de recursos desde los niveles centrales para atender problemas de la pobreza urbana y déficits de infraestructura. De igual manera, se han venido implementando en forma incipiente nuevas formas de gobierno y gestión pública, con el objeto de lograr mayores niveles de eficacia y legitimidad de la acción gubernamental en el ámbito local urbano.

En este escenario, el proceso de gobernar ciudades el desafío de atender nuevos conflictos y retos generados por la creciente diversidad de demandas y la mayor interdependencia entre el Estado y la sociedad. Nuevas modalidades de política y gestión urbana, acordes con los cambios planteados y un contexto de mayor complejidad, se vuelven necesarias con el fin de compatibilizar intereses diversos y generar estrategias sociales e institucionales más consolidadas. No obstante, esto estará permeado por la impronta políticoideológica de los modelos de intervención predominantes y el conjunto de intereses en juego. 


\section{BALANCE E IMPLICANCIAS DE LA ORGANIZACIÓN POLÍTICA Y ADMINISTRATIVA DE LAS CIUDADES EN EL CONTEXTO ARGENTINO}

En los últimos veinte años las grandes transformaciones estructurales por las que atravesó la Argentina (procesos de apertura y globalización, privatizaciones, descentralización) afectaron de manera particular a las ciudades y la escala local de gobierno. En un escenario de reforma del Estado y ajuste fiscal, la transferencia de funciones desarrolladas en la década de los noventa desde el gobierno nacional a las gobiernos provinciales y municipales supuso una delegación "hacia abajo" de la crisis del nivel central. Esto determinó, por una parte, el incremento de las intervenciones desarrolladas por las instancias territoriales de gobierno (con una abultada agenda para los gobiernos locales de las ciudades) y, por otra parte, un aumento de las demandas y expectativas de la población local hacia los municipios (Arroyo, 1997; Madoery, 2001 y 2005, Iturburu, 2000 y 2007, Carmona, 2006 y 2012).

Las instancias locales debieron asumir de "hecho" nuevas responsabilidades de gestión en temas tales como empleo, medio ambiente, asistencia social y promoción económica, sin contar en la mayor parte de los casos con los recursos y atribuciones para ello. Los cambios producidos llevaron a las autoridades locales a una estrategia de mayor cercanía con la ciudadanía, a la vez que aumentaron las exigencias de la misma hacia este primer eslabón del Estado en el territorio. Esta dinámica trajo aparejada además la discusión respecto a la importancia de redefinir los modelos tradicionales de gestión local y generar nuevos tipos de intervención en las ciudades.

Los gobiernos locales, identificados por lo general con la esfera municipal, han tenido tradicionalmente un papel secundario respecto a los distintos medios y competencias para favorecer el desarrollo social y económico. Las características de un sistema institucional históricamente enraizado en el plano de recursos y atribuciones en la esfera nacional y provincial, determinó un esquema municipal acotado en grandes líneas a la obra pública y el suministro de servicios urbanos básicos (recolección de residuos sólidos, alumbrado público y mantenimiento urbano.). En efecto, en un país federal como la Argentina el peso y protagonismo que adquiere la esfera provincial con un sistema propio de organización municipal es muy relevante, puesto que existen en el país 23 regímenes municipales distintos (a ello se suma la Ciudad Autónoma de Buenos Aires, con un estatus de "cuasi-provincia" y un sistema interno de Comunas).

A este cuadro de complejidad institucional, se le suma la fuerte diferencia en escala, capacidades y perfil de 
desarrollo que hay entre los gobiernos locales. En nuestro país existen aproximadamente 1.165 municipios y 1.086 gobiernos locales dependientes de la jurisdicción provincial sin estatus municipal. A ello, se le suman gobiernos locales conformados por municipios -comisiones de fomento, juntas vecinales-, que vienen a constituir en la práctica un cuarto nivel de gobierno, y cuya cantidad se estima en más de 300. De este modo, el número total en Argentina supera los 2.500 gobiernos locales, entre los cuales sobresalen las ciudades de más de 100 mil habitantes (urbes intermedias y áreas metropolitanas) que concentran alrededor de dos tercios de la población total. Sin embargo, la mayoría de los gobiernos locales tienen menos de 10 mil habitantes. Esto conforma un escenario local urbano muy heterogéneo con problemáticas y posibilidades de desarrollo distintas en relación a las trayectorias históricas, recursos disponibles, estrategias y condiciones técnicas y organizativas de partida (García Delgado, 1997; Arroyo, 2001; Iturburu, 2012).

Un análisis de la participación del gasto público por jurisdicción aparece como un indicador ineludible para examinar el perfil que se le define a los municipios en nuestro país y dar cuenta de la evolución de esta variable a lo largo de las últimas décadas. En primer lugar, es posible apreciar la disminución relativa del gasto del gobierno nacional, si tienen en cuenta los valores extremos de la serie, que evidencian el traspaso de responsabilidades de gasto desde el ámbito federal a las jurisdicciones subnacionales, en especial al nivel provincial. En segundo lugar, un aumento del gasto público municipal que se duplica de la década del ' 80 a la del '90, pero que luego se mantiene estable y hasta con una leve disminución en la actualidad

Figura 1. Evolución del gasto público por jurisdicción

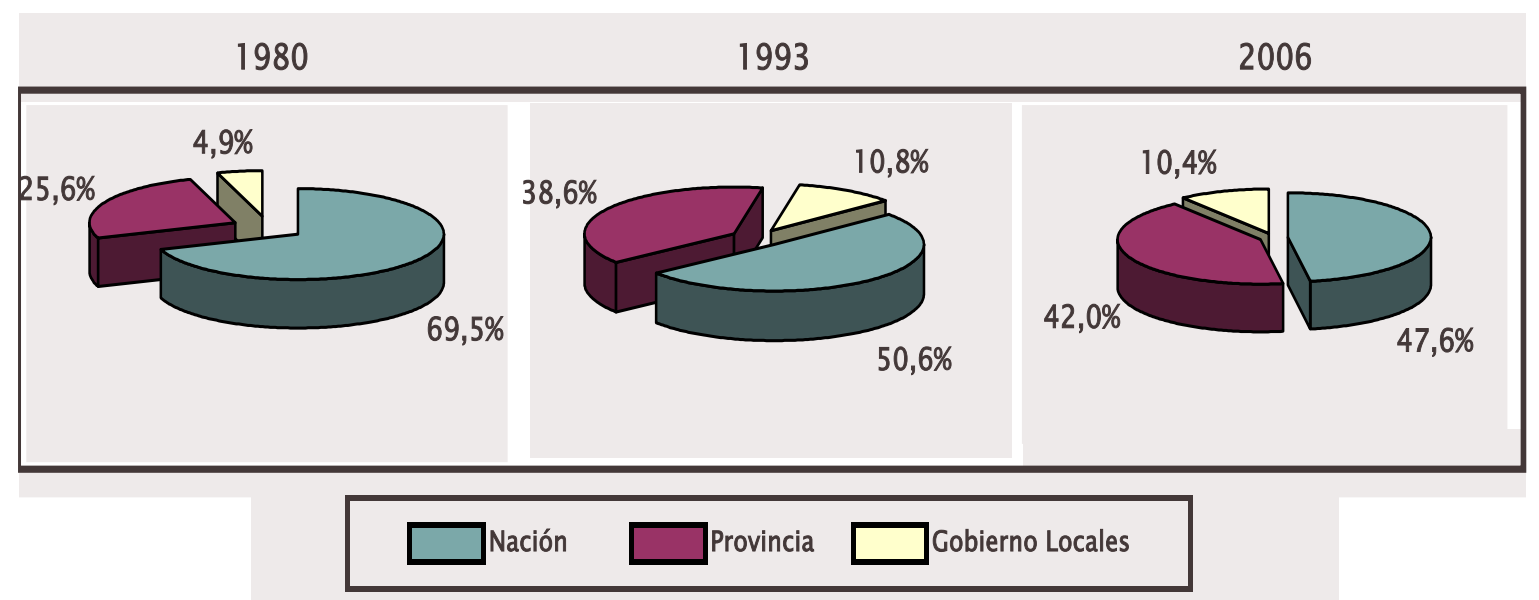

Fuente: Elaboración propia en base a datos del Ministerio de Economía Obras y Servicios Públicos/ Ministerio de Economía y Finanzas Públicas 
En estos últimos años, las transferencias realizadas desde los niveles superiores de gobierno hacia los municipios dejan entrever el mayor peso que van adquiriendo los gobiernos locales y las ciudades en materia de actuación. En efecto, según se puede apreciar en las figuras 2 y 3 , el incremento de las transferencias tanto desde el nivel provincial como nacional es muy significativo en el último período. En el primer caso, las transferencias provinciales hacia los municipios por objeto del gasto aumentan de 2002 a 2009 más de un 126\%. Del mismo modo, y con un papel más activo del Estado Nacional y mayores recursos, las transferencias directas hacia los municipios se elevan casi un $240 \%$ para el mismo período. Estas transferencias de la última década obedecieron así a una estrategia institucional deliberada de traspaso de funciones básicas y una forma de compensar, en algunos casos de forma discrecional y/o con mayor control de los niveles superiores -sobre todo de nación-, la escasa participación real de los gobiernos locales en el gasto público total.

Figura 2. Transferencias de las Provincias a Municipios. Período 2002-2009

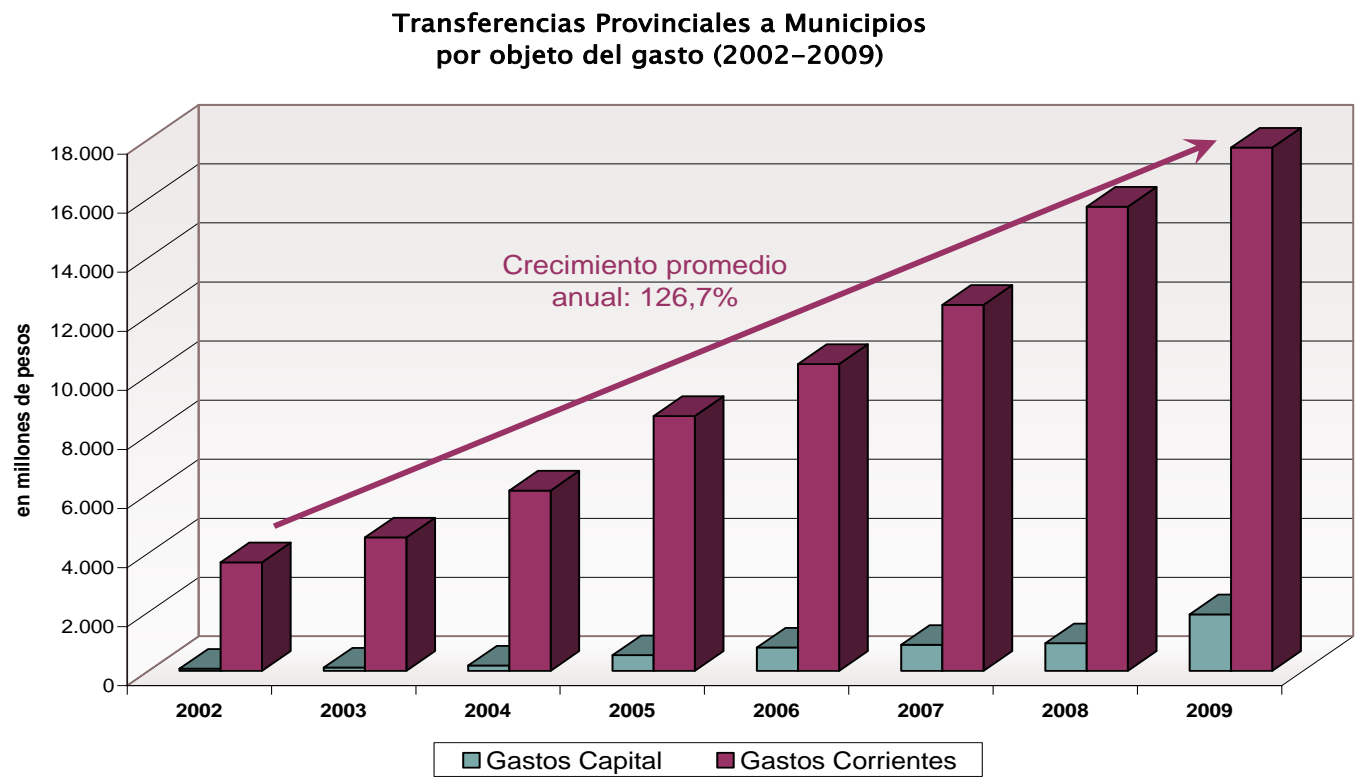

Fuente: Elaboración propia en base a datos del Ministerio de Economía y Finanzas Públicas 
Figura 3. Transferencias de la Nación a Municipios. Período 2002-2009

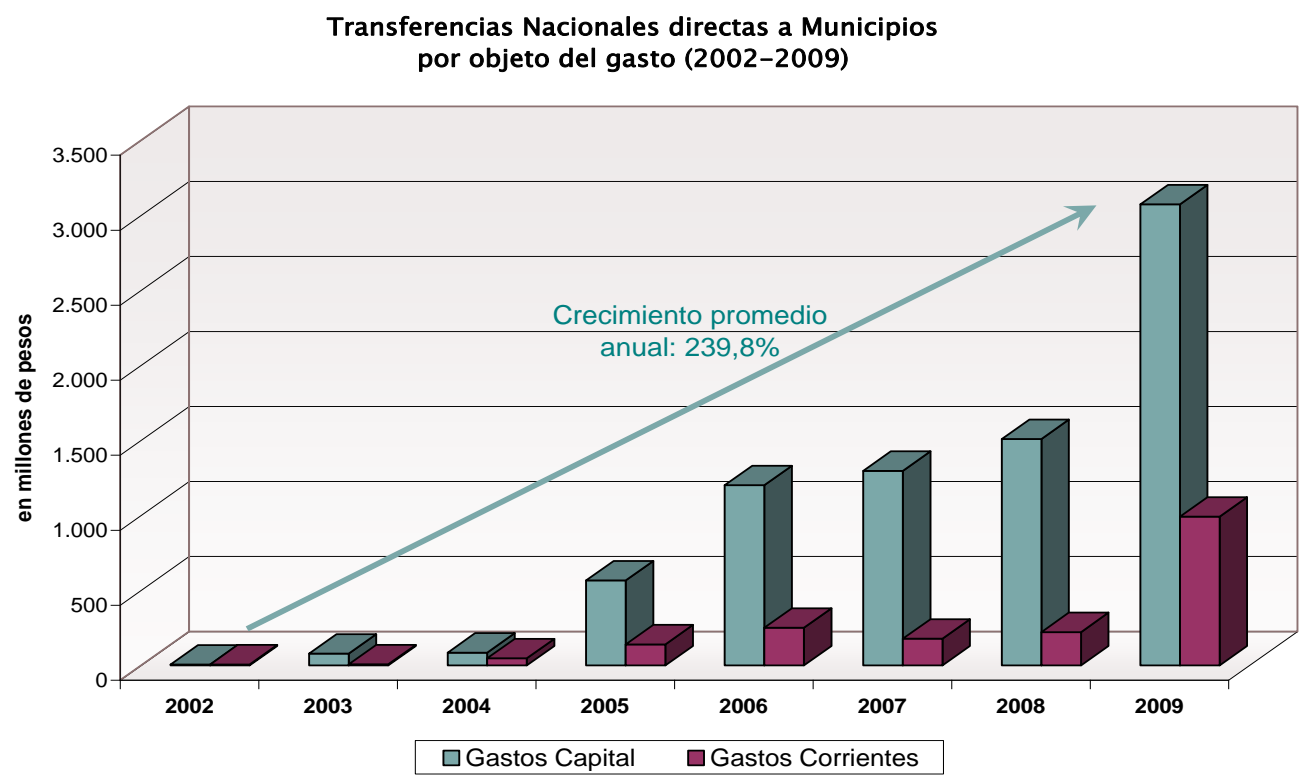

Fuente: Elaboración propia en base a datos del Ministerio de Economía y Finanzas Públicas

En definitiva, los gobiernos locales asumen nuevas responsabilidades para dar cuenta de los problemas y demandas de diferente envergadura que acontecen en las ciudades. Esta problemática, no obstante, muestra fuertes niveles de variabilidad al considerar factores claves como el tamaño de la población, la capacidad económica e institucional de los ámbitos locales urbanos.

\section{El fenómeno urbano en Argentina}

La Argentina, al igual que otros países de la región, está a la vanguardia del fenómeno mundial de concentración poblacional en ciudades. Según datos censales de 2010, la población supera los 40 millones de personas y 9 de cada 10 habitantes vive en ciudades (92\%). Este valor es sumamente alto a nivel internacional y según estimaciones de la CEPAL equivalente a naciones vecinas como Uruguay y Venezuela, y cercano a Brasil y Chile. El proceso de urbanización argentino se ha desplegado de manera acelerada desde las primeras décadas del siglo XX, con una tendencia clara desde los años '50 vinculada a la reducción de la población rural en términos absolutos. En las últimas décadas, el crecimiento de dicho proceso ha sido menor dado el alto nivel de urbanización alcanzado. No obstante, el porcentaje de población urbana varía según las regionales y es esperable que en las menos urbanizadas la dinámica continúe, especialmente en el Noroeste y el Nordeste argentino. 
Es importante considerar también el proceso de crecimiento urbano desplegado a los largo de los años. En estos términos, el estrato de ciudades de mayor crecimiento ha sido el que se ubica entre los 50 mil y 500 mil habitantes que concuerda generalmente con las ciudades intermedias, con un papel de intermediación que suelen asumir en el plano de la organización territorial. Del mismo modo, se observa a nivel censal un menor ritmo de crecimiento de las ciudades más grandes, en particular de Buenos Aires como conjunto metropolitano, con una pérdida de primacía en el conjunto.

En consecuencia, el patrón de crecimiento urbano reciente plantea diversas situaciones. Por un lado, grandes ciudades pampeanas o de la zona central del país con descenso de crecimiento y ciudades intermedias en el Nordeste, Noroeste y Patagonia con alto crecimiento. Por otra parte, grandes ciudades fuera de la región pampeana y ciudades pampeanas de mediano tamaño con crecimiento intermedio. Se aprecia así un proceso simultáneo que combina desconcentración geográfica de la población urbana y de los estratos de mayor población (Plan Estratégico Territorial, 2011).

\section{Marco político-institucional de los gobiernos locales}

La definición del gobierno local en clave municipal ha sido fruto de debate a lo largo del tiempo. El artículo $5^{\circ}$ de la Constitución Nacional Argentina establece que: "Cada provincia dictará para sí una Constitución bajo el sistema representativo republicano...que asegure su administración de justicia, su régimen municipal, y la educación primaria. Bajo estas condiciones el Gobierno federal garante a cada provincia el goce y ejercicio de sus instituciones". Del mismo modo, el artículo $123^{\circ}$, determina claramente la autonomía municipal cuando plantea que: "Cada provincia dicta su propia Constitución, conforme a lo dispuesto en el artículo $5^{\circ}$ asegurando la autonomía municipal y reglando su alcance y contenido en el orden institucional, político, administrativo, económico y financiero".

A partir de encuadre, cada constitución provincial define su régimen municipal. El parámetro más utilizado para conformar un municipio es la población, en algunos pocos casos se establecen o añaden otras condiciones (por ejemplo, ser partido o departamento), o directamente no se especifican requisitos. La mayoría de las constituciones establece entre 500 y 2.000 habitantes como población mínima. Aunque estos pequeños municipios presentan ventajas en términos de representación, generalmente utilizan sus recursos escasos para los gastos administrativos fijos. De esta forma, resulta complicado que gran parte de las constituciones provinciales establezcan mínimos poblacionales muy bajos, lo cual hace altamente dificultosa la tarea de gobernar dado la escasez de recursos y capacidades de estos 
territorios. La autonomía municipal se haya ampliamente extendida y consagrada en las cartas magnas provinciales. Sin embargo, tres de las principales provincias argentinas (Buenos Aires, Santa Fe y Mendoza), que reúnen casi la mitad de la población, no tienen autonomía para sus municipios, junto a una situación caracterizada por una regulación limitada en gran parte de las provincias que sí la reconocen.

Una gran proporción de las constituciones muestra también poca claridad en la delimitación territorial de los municipios y en muchos casos, delegan en el Poder Legislativo provincial la fijación definitiva de límites. En el funcionamiento práctico, se adhiere de manera equilibrada al sistema de ejidos colindantes y no colindantes. Ello genera graves problemas a los grupos humanos ubicados en los resquicios entre municipios, que quedan substraídos de toda protección y asistencia estatal (Iturburu, 2000).

Las constituciones en general exhiben también distintos sistemas de determinación de las competencias. Las variantes comprenden la definición de un tope mínimo y máximo, la determinación de una escala incremental o la inclusión de excepciones, tales como ser la capital de la provincia. Por último, las condiciones para que un municipio dicte su carta orgánica son muy dispares y los casos extremos son indicativos de esta afirmación-desde un mínimo de 1.000 habitantes hasta 30.000-. Hasta el momento han dictado su carta orgánica 150 municipios de 14 provincias de las 20 que reconocen la autonomía municipal (Iturburu, 2012).

\section{Gobierno de las ciudades y heterogeneidad}

La fuerte heterogeneidad y disparidad de situaciones en las provincias argentinas se refleja en el plano de los gobiernos locales y las ciudades. El diseño institucional municipal definido por cada jurisdicción provincial determina diferentes modalidades y requisitos de conformación, definición del territorio, forma de gobierno, recursos y atribuciones, entre otros elementos. Una cuestión que se presenta en diversas provincias es la presencia de una ley orgánica más antigua que la constitución vigente, lo que genera confusión respecto del diseño desarrollado y, en algunos casos, un fuerte choque de criterios. Otro punto, se vincula con las diferencias demográficas de los gobiernos locales desde municipios muy grandes, incluso con mayor población que gran parte de las provincias, hasta municipios muy pequeños. La media nacional se ubica en los 2.000 habitantes, si bien el promedio general es menor a los 20.000 habitantes.

De este modo, el municipio argentino más poblado según el Censo 2010 es La Matanza en la Provincia de Buenos Aires, con casi 1,8 millones de habitantes y más población que 20 de 
las 23 provincias argentinas. En este marco, se ha iniciado hace algún tiempo un proceso de discusión sobre la regionalización del gobierno provincial con escasos resultados hasta el momento. La provincia de Córdoba, por su parte, presenta el segundo municipio más poblado del país en la ciudad capital, con más de 1,3 millones de habitantes, y muestra también los problemas de los municipios muy pequeños, 427 gobiernos locales, de los cuales una tercera parte presenta menos de 500 habitantes. En 2004, se buscó generar allí un nuevo proceso de regionalización en el $85 \%$ de los municipios provinciales con avances dispares. Esta tendencia de municipios muy reducidos se repite también en otras provincias, dando lugar a casi un centenar de municipios argentinos con menos de 100 habitantes.

Es posible apreciar así un proceso variado marcado por distintas dinámicas. En primer lugar, la presencia de gobiernos locales de menos de 1.000 habitantes que representan más de un tercio del total de municipios argentinos y el 1\% del conjunto de población total. En segundo término, municipios de más de 150 mil habitantes que suponen más del $2 \%$ del total de gobiernos locales y concentran la mitad de la población del país. Ello determina un cuadro caracterizado por el desarrollo de metrópolis y aglomerados urbanos, dónde la urbanización alcanza el territorio de varios municipios (por ejemplo, las ciudades de Buenos Aires y Córdoba); la concentración poblacional en capitales o ciudades más importantes de cada provincia; la división y creación de gobiernos locales muy reducidos; y el despliegue de nuevas asociaciones municipales y procesos de regionalización.

Este escenario de heterogeneidad se aprecia también a nivel fiscal. Cómo destaca López Acotto (2005:1): “Una primera limitación fuerte que tienen los municipios en Argentina es la muy escasa participación tributaria de sus ingresos. Es decir que una de las herramientas más importantes para la búsqueda de la equidad es ajena a los municipios. Sólo el 2,8\% del total de ingresos municipales es de origen tributario....Esta situación también se refleja en la enorme dispersión existente en cuanto a la autonomía financiera municipal que va de un valor elevado de un $72 \%$ de recursos propios en los municipios de la provincia de Chubut a un exiguo $8 \%$ en los municipios de la Provincia de La Rioja".

Esto plantea un contexto de gran diversidad entre provincias, en cuanto a las distancias que cada una tiene en relación al gasto municipal per cápita. Ello destaca un escenario de marcadas desigualdades en cuanto al nivel de desarrollo por jurisdicción, con fuertes diferencias entre provincias y hacia el interior de cada una de ellas. Se identifican entonces, según resalta Iturburu (2012), provincias de gasto municipal per cápita alto (en las que imperan las provincias mineras/ petrolíferas y alguna de la pampa húmeda), que se posicionan por encima del promedio nacional; provincias de gasto municipal per cápita medio (como por ejemplo, Entre Ríos, Río Negro, Buenos Aires y Santa Fe), que se ubican cerca del promedio nacional; y 
provincias de gasto municipal per cápita bajo (donde predominan las provincias del noroeste, noreste y las cuyanas), por debajo del promedio nacional. La disparidad fiscal entre los municipios se explica también en relación a la situación económica de los residentes y su capacidad contributiva, como respecto a la descentralización del gasto.

\section{Gestión pública, políticas locales y nueva agenda de las ciudades}

A diferencia de los niveles superiores de gobierno, los municipios presentan estructuras orgánicas generalmente más informales y sujetas a la decisión del intendente para la conformación o disolución de una dependencia municipal. En algunos casos a las dificultades para constituir un aparato burocrático en términos weberianos, se suman estilos de liderazgo político local muy centralizadores de la decisión y poco abiertos a nuevas prácticas administrativas y democráticas por parte de las autoridades. Ello determina que las administraciones públicas municipales sean muy dinámicas y cambiantes en su organigrama interno. Predomina en términos funcionales y más allá de las diferencias de las ciudades, un esquema organizacional con diferentes dependencias en el área de gobierno, hacienda, servicios y obras públicas y asistencia social. En general, la división en gobiernos locales pequeños se realiza más en términos personales (el intendente y algún secretario) y en municipios más grandes y urbanizados, alcanza una mayor institucionalización con diversas secretarías.

Del mismo modo, es posible distinguir distintos modelos políticos de gobierno de la ciudad con un anclaje más mercantil-privado (por ejemplo, la Ciudad Autónoma de Buenos Aires o el municipio de Tigre en la Provincia de Buenos Aires) y más centrado en un enfoque de derechos y la ciudadanía (por ejemplo, la ciudad de Rosario en la Provincia de Santa Fe o el municipio de Morón en la Provincia de Buenos Aires). Si bien estos modelos no se expresan en forma pura, aparecen sesgos muy marcados en los gobiernos las ciudades, con una fuerte tensión en términos del mercado de suelo, la vivienda, el desarrollo urbano y los impactos sociales de estas dinámicas.

Un análisis más puntual de las políticas nos muestra que las iniciativas desplegadas en las ciudades a partir de los años '90 surgieron en sus comienzos como comportamientos claramente defensivos a los procesos de cambio, y con el tiempo fueron dirigiéndose a discutir cuestiones más amplias vinculadas con la redefinición del rol y el tipo de intervención de los gobiernos locales. Esta última tendencia puede apreciarse en la búsqueda de un anclaje local para diversas políticas nacionales (y también provinciales, aunque con menor fuerza) en el área económica, social y de infraestructura, la mejora de los procesos administrativos y de gestión 
internos, la extensión de la descentralización local, el planeamiento estratégico y el presupuesto participativo, entre otras iniciativas.

En este escenario, aunque los gobiernos locales no han logrado en general materializar una autonomía institucional que se traduzca en una genuina capacidad de tomar decisiones y de extraer o asignar recursos -factor determinante en diversos contextos-, es importante destacar el surgimiento de nuevas intervenciones y articulaciones multiactorales con el objeto de dar respuesta a una gran diversidad de cuestiones que aparecen en el plano territorial y que revelan un cambio respecto a décadas pasadas (Cravacuore, llari y Villar, 2004; Carmona, 2006 y 2012).

Este proceso relacionado a nuevos modelos de gobierno y gestión local o gobernanza es aún incipiente y requiere para su afianzamiento de cambios, no únicamente en el plano local, sino también en la órbita nacional y provincial, de modo de poder impulsar el desarrollo de esos territorios. Un conjunto diverso de actividades donde el Estado local articula con otras instituciones y actores de la ciudadanía en general, aparece cada vez más con mayor fuerza y se refleja en la implementación de diversas políticas públicas, asociaciones de distinto tipo y vinculaciones público-privadas.

En efecto, a las tareas tradicionales cumplidas por los gobiernos en las ciudades (con eje, en los servicios urbanos básicos) se han venido incorporando nuevas funciones vinculadas con: el desarrollo social, la promoción económica, la generación de empleo, la salud, el despliegue de obras de infraestructura y viviendas, el fortalecimiento de instalaciones educativas y tecnológicas, el cuidado del medio ambiente, el desarrollo cultural, la defensa del patrimonio histórico, la resolución de conflictos y reivindicación de derechos de nuevo tipo, la seguridad y el establecimiento de diferentes canales de participación ciudadana. Los condicionantes del contexto y la mayor cercanía de los ciudadanos con los gobiernos locales hacen que las instancias municipales deban responder a estos nuevos requerimientos, que en general superan sus competencias y atribuciones ante una mayor expectativa y demanda de la población.

Las dificultades que muestran los gobiernos locales en el escenario argentino se hacen aún más evidentes en las ciudades metropolitanas. Estos centros urbanos, que concentran más de la mitad de la población del país, no son en la práctica instancias directas de gobierno que puedan dar cuenta de los problemas. Su desarrollo está sujeto a un conjunto variado de gobiernos locales (municipios) y el fuerte protagonismo del nivel provincial y nacional en su funcionamiento, con características particulares según los casos. La ciudad metropolitana argentina, de este modo, no conforma una unidad institucional concreta en términos de representación y acumulación política. En primer término, 
no hay vinculación entre el aparato político de toma de decisiones y la sociedad en un metropolitano, puesto que no tiene control sobre los votos para elegir autoridades 0 legisladores. En segundo lugar, la dinámica de los actores políticos no tiene una operatoria y sustento de tipo metropolitano. Esto se hace palpable a partir del hecho que la ciudad como conjunto no participa en la definición de políticas y carece de los recursos indispensables para influir en el plano federal o nacional, y en gran medida, en el provincial (Pírez, 2005 y 2008; Badía, Carmona y Soldano, 2008; Carmona, 2012).

En estos términos, las ciudades metropolitanas aparecen como realidades políticoinstitucionales fragmentadas donde las decisiones quedan sujetas a los gobiernos municipales involucrados y, fundamentalmente, a los gobiernos provinciales. Estas ciudades presentan gran relevancia en las relaciones políticas establecidas a nivel provincial, como es el caso de las capitales o ciudades de gran envergadura (por ejemplo, Rosario en la Provincia de Santa Fe o Río Cuarto en la Provincia de Córdoba). Las relaciones predominantes son así de competencia entre los gobiernos locales que forman parte del área metropolitana y de dependencia respecto a las decisiones derivadas desde la esfera provincial.

En un marco donde los gobiernos municipales son fuertemente dependientes desde lo financiero y tienen escasas capacidades y atribuciones para desarrollar un papel protagónico, los gobiernos provinciales controlan los principales "clivajes" para hacer política en el plano territorial. Esto determina mayormente un sistema de relaciones radiales entre la provincia y los municipios, mediado por el aporte de recursos a nivel central y el apoyo de los municipios a los mecanismos de acumulación política provincial (votos para las elecciones, apoyo en internas partidarias y para campañas electorales), lo cual limita la posibilidad de generar instancias de cooperación y coordinación entre las instancias locales y pensar en una plano metropolitano más sistémicamente.

Aunque los niveles de crecimiento de las ciudades metropolitanas disminuyen en las últimas décadas, su protagonismo es muy marcado en la escena nacional y presentan diferencias importantes entre ellas. Por una parte, la Región Metropolitana de Buenos Aires (RMBA) constituye una realidad en sí misma que difiere de las otras dos grandes zonas metropolitanas del país que tienen más de un millón de habitantes (Córdoba y Rosario) y, mucho más, respecto de las ciudades capitales del interior que tienen niveles poblaciones más

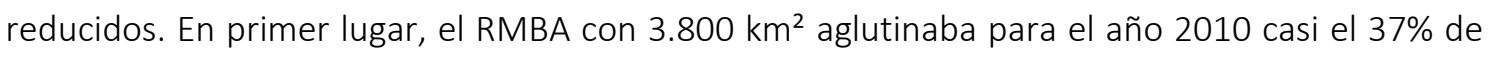
la población nacional (14,8 millones de personas), de los cuales casi 2,9 millones habitaban en la Ciudad Autónoma de Buenos Aires y los 12,8 millones restantes en municipios del denominado Conurbano Bonaerense y alrededores. Este territorio, eje central del país, participaba en aproximadamente el 50\% del PBI nacional. 
Estos aspectos plantean entonces diversos problemas y desafíos para las áreas metropolitanas. Se visualizan así inconvenientes de tipo político-institucional ante la diversidad de niveles gubernamentales implicados en el territorio tanto en términos de planificación como de actuación. La participación de distintos gobiernos municipales, junto a uno o más gobiernos provinciales según el caso y el fuerte predomino que tiene el gobierno federal en los últimos años -en términos de acciones y transferencias de recursos que se direccionan en muchas ocasiones directamente a las instancias locales sin pasar por la órbita provincial-, hacen evidente una realidad metropolitana compleja y que demanda una mayor articulación institucional, con algunos avances en términos de visualizar los problemas en diferentes ejes y evaluar alternativas de mayor colaboración (por ejemplo, en materia ambiental, socioeconómica, infraestructura, seguridad y transporte).

\section{CONSIDERACIONES FINALES}

El hacer frente a problemas de gran envergadura y complejidad aparece como uno de los principales desafíos que tienen los centros urbanos y áreas metropolitanas en la actualidad. De esta forma, las ciudades se convierten en portadoras de distintos cambios y contradicciones producto de los procesos de transformación en curso. En efecto, estos territorios constituyen ámbitos significativos para el crecimiento económico y la gobernabilidad. Por otra parte, fenómenos tales como la pobreza urbana, la segregación socioespacial y desequilibrios de distinta naturaleza adquieren mayor intensidad en este tipo de espacios.

La situación existente en gran parte de las ciudades argentinas muestra en general gobiernos con escasas acciones innovadoras. Para ello confluyen, debilidades administrativas e institucionales arraigadas en el tiempo, problemas de coordinación intergubernamental, liderazgos políticos poco trasformadores e inconvenientes en el plano de la articulación con los actores sociales y la canalización de sus demandas. El análisis de estos factores, sin embargo, varía según la historia y particularidades de cada urbe.

El contexto urbano argentino presenta así diferentes dificultades: la falta de un sistema de políticas articulado e institucionalizado para el estímulo pleno de lo "local" y un desarrollo urbano más planificado e inclusivo; condicionamientos de tipo económico y social que persisten pese a las mejoras de los últimos años; cierta saturación en la capacidad de gestión de los gobiernos locales; debilidad de los actores (públicos, privados y sociales) para propiciar iniciativas endógenas más proactivas y transformadoras; heterogeneidad y complejidad de situaciones territoriales a lo largo del país; y la ausencia de mecanismos de medición, impacto y aprendizaje en la gestión. 
En las ciudades argentinas se espera por lo general que el gobierno local pueda responder al conjunto de cuestiones y problemas que acontecen en el territorio, sin considerar el nivel de gobierno que tiene las atribuciones para dar cuenta de ello. Esta cuestión plantea la necesidad cada vez más imperiosa que los diseños institucionales puedan dar cuenta de las diferencias de escala de las ciudades asignando mayores recursos, funciones y autonomía a los municipios más grandes (con el despliegue de instancias metropolitanas y sectoriales que contemplen el territorio real), además de promover un mayor grado de coordinación, control, cooperación y/o regionalización de los gobiernos locales más débiles y pequeños.

Se requiere así del apoyo de los gobiernos provinciales y su legislación en la generación de mayores márgenes de autonomía municipal, descentralización de recursos tributarios para las nuevas funciones asumidas y acompañamiento de las administraciones locales en términos de capacitación, formación de recursos humanos e impulso a la innovación de la gestión tanto en términos políticos como técnicos. Se hace relevante también contar con una conducción política de la ciudad capaz de planificar la gestión que pretende llevar a cabo, esto es, gobiernos con una agenda precisa para resolver los problemas más significativos de sus comunidades. Del mismo modo, el Estado nacional al tener un rol activo en el despliegue de políticas y transferencias de recursos hacia los gobiernos locales aparece como un actor determinante en pos de lograr una mayor integración del territorio y planificación en diversos ejes estratégicos.

En este sentido, se torna prioritario establecer marcos de intervención intergubernamental que contemplen desde niveles básicos de coordinación territorial, hasta la creación de nuevas lógicas y formas de actuación que incorporen atribuciones especiales para ciertos gobiernos locales y áreas metropolitanas como la incorporación de mecanismos más participativos y de apertura de las decisiones. El reto central en el contexto argentino pasa entonces por promover políticas locales urbanas adecuadas, articuladas con el nivel nacionalprovincial o regional ampliado e internacional según el caso y los actores sociales, de modo de llevar a cabo acciones e intervenciones más efectivas, legítimas y estimular proyectos colectivos superadores. En consecuencia, nuevos esquemas de política y gestión urbana se vuelven necesarios a fin de atender las dificultades presentes y generar estrategias más articuladas y consolidadas en un escenario más dinámico y cambiante como el que caracteriza a nuestras sociedades en la actualidad.

\section{BIBLIOGRAFÍA}

ARROYO, Daniel. Los ejes centrales del desarrollo en la Argentina. FLACSO-Argentina. Mimeo. 2001. 
Estilos de gestión y políticas sociales municipales en Argentina. En GARCíA DELGADO, Daniel (Comp.) Hacia un nuevo modelo de gestión local. Municipio y sociedad civil en Argentina. Ed. FLACSO, CBC y Universidad Católica de Córdoba. Buenos Aires. 1997.

BADÍA, Gustavo; CARMONA, Rodrigo y SOLDANO, Daniela. El orden político a nivel local. Estado, sociedad y políticas públicas en municipios de la Región Metropolitana de Buenos Aires. En BADÍA, Gustavo y CARMONA, Rodrigo (Comp.) La Gestión Local en Argentina: situación y perspectivas. Universidad Nacional de General Sarmiento. Los Polvorines-Buenos Aires. 2008.

BLANCO, Ismael y SUBIRATS, Joan. ¿Existen territorios socialmente excluyentes? Contra lo inexorable. En BLANCO, Ismael; FLEURY, Sonia y SUBIRATS, Joan (Coord.) Innovaciones locales ante inseguridades globales: los casos de Brasil y España. Colección Interrogar la Actualidad, Fundación CIDOB. Barcelona. 2008.

BLANCO, Ismael y GOMÁ, Ricard. Del gobierno a la gobernanza. Oportunidades y retos de un nuevo paradigma. Polítika Revista de Ciencias Sociales. 2, 1-27.2006.

BORJA, Jordi y CASTELLS, Manuel. Local y Global. La gestión de las ciudades en la era de la información. United Nations for Human Settlements (Habitat). Taurus Pensamiento. Madrid.1997

MENDOZA, Enrique Cabrero. Acción pública y desarrollo local. Fondo de Cultura Económica. México.2005.

CARMONA, Rodrigo. El debate sobre nuevos estilos de gobierno en ciudades argentinas. CICCUSUniversidad Nacional de General Sarmiento. Buenos Aires. 2012.

Nuevas formas de gobierno y gestión pública en el escenario local. Algunos ejes de debate para el estímulo de procesos de desarrollo, en ROFMAN, Adriana y VILLAR, Alejandro. (Comps.) Desarrollo local. Una revisión crítica del debate. Universidad Nacional de Quilmes Universidad Nacional de General Sarmiento, Espacio Editorial, Buenos Aires. 2006.

CARRIÓN M. Fernando. El desafío político de gobernar la ciudad. Revista Nueva Sociedad, N ${ }^{\circ}$ 212, noviembre-diciembre de 2007. Disponible en: https://www.nuso.org/revista.php?n=212.

CASTELLS, Manuel. La Era de la Información. Economía, Sociedad y Cultura, Vol.1 La Sociedad Red, Madrid: Alianza. 1996.

CRAVACUORE, Daniel; ILARI, Sergio y VILLAR, Alejandro. La articulación de la gestión municipal. Actores y políticas. Universidad Nacional de Quilmes. Buenos Aires. 2014.

ITURBURU, Mónica. Los municipios. En CAO, Horacio y ABAL MEDINA, Juan Manuel (Comps.) Manual de la nueva administración pública argentina. Ariel. Buenos Aires. 2012.

Modernización en los municipios argentinos. En: CRAVACUORE, Daniel e ISRAEL, Ricardo (comp.) Procesos políticos municipales comparados en Argentina y Chile (1990-2005). Editorial de la Universidad Nacional de Quilmes - Universidad Autónoma de Chile. Buenos Aires. 2007.

Municipios argentinos. Fortalezas y debilidades de su diseño institucional. INAP. Buenos Aires. 2000.

GARCÍA DELGADO, Daniel. Nuevos escenarios locales. El cambio de modelo de gestión. En GARCÍA DELGADO, Daniel (comp.) Hacia un nuevo modelo de gestión local. Municipio y Sociedad Civil en Argentina. FLACSO, UBA-CBC y Univ. Católica de Córdoba. Bs. As. 1997. 
GRAÑA, Francois. Diálogo social y gobernanza en la era del "Estado Mínimo". Montevideo: Cintefor/OIT. 2005.

LÓPEZ ACOTTO, Alejandro. Los municipios y el desarrollo con equidad. Ponencia presentada en El Plan Fénix en vísperas del segundo centenario. Una estrategia nacional de desarrollo con equidad. Buenos Aires, 02-05 de Agosto de 2005.

MADOERY, Oscar. (2005). La "primera generación" de políticas locales de desarrollo en Argentina: Contexto, características y desafíos. CEDET. Disponible en http://www.cedet.edu.ar/Archivos/Bibliotecas

El valor de la política en el desarrollo local. En VÁZQUEZ BARQUERO, Antonio y MADOERY, Oscar (comp.) Transformaciones globales, instituciones y políticas de desarrollo local. Homo Sapiens Ediciones, Rosario. 2001.

Plan Estratégico Territorial: Avance II. Argentina Urbana - Lineamientos Estratégicos para una Política Nacional de Urbanización. Ministerio de Planificación Federal, Inversión Pública y Servicios, Buenos Aires. 2011.

PÍREZ, Pedro. Gobernabilidad metropolitana en la Argentina. En BADÍA, Gustavo y CARMONA, Rodrigo (Comp.) La Gestión Local en Argentina: situación y perspectivas. Universidad Nacional de General Sarmiento. Los Polvorines-Buenos Aires. 2008.

El desafío de la Gobernabilidad Metropolitana en la Argentina. Ponencia presentada en el VII SEMINARIO RedMuni. 15 y 16 de septiembre de 2005. UNGS-INAP. 2005.

Descentralización, gobierno colegiado y gobernabilidad urbana. Algunas reflexiones. En Escolar, M.; Badía, G. y Frederic, S. (Edit.) Federalismo y descentralización en grandes ciudades: Buenos Aires en perspectiva comparada. Prometeo. Buenos Aires. 2004.

Gobernabilidad urbana en una ciudad metropolitana fragmentada. Ponencia en el VI Seminario Internacional de la Red Iberoamericana de Investigadores sobre Globalización y Territorio. Rosario: Mayo. 2001.

SASSEN, Saskia. La Ciudad Global: Nueva York, Londres, Tokio. EUDEBA. Buenos Aires. 1999. Cities in a world economy. California: Pine Forge Press (A Sage Publications Company).

VILLANUEVA, Luis Fernando Aguilar. El aporte de la política pública y la Nueva Gestión Pública a la gobernanza. XI Congreso Internacional del CLAD sobre la Reforma del Estado y de la Administración Pública. Sto. Domingo, Rep. Dominicana, 30 oct. - 2 nov. 2007.

Trabalho enviado em 07 de novembro de 2014.

Aceito em 05 de dezembro de 2014. 